\title{
Molecular Diagnosis in House Dust Mite-Allergic Patients Suggests That Der p 23 Is Clinically Relevant in Asthmatic Children
}

\author{
Jiménez-Feijoo R¹, Pascal M², Moya R³, Riggioni C, Domínguez 0', Lózano J', Álvaro-Lozano M', \\ Piquert $\mathrm{M}^{1}$, Machinena $\mathrm{A}^{1}$, Folque $\mathrm{M}^{1}$, Dias $\mathrm{M}^{1}$, Carnés ${ }^{3}$, Plaza $\mathrm{AM}^{1}$ \\ 'Department of Pediatric Allergy and Clinical Immunology, Hospital Sant Joan de Déu, Universitat de Barcelona, Esplugues de Llobregat, \\ Barcelona, Spain \\ ${ }^{2}$ Immunology Department, CDB, Hospital Clínic de Barcelona, IDIBAPS, Universitat de Barcelona, Barcelona, Spain; ARADyAL, \\ Instituto de Salud Carlos III, Madrid, Spain \\ ${ }^{3}$ REtD Allergy and Immunology Unit, Laboratorios LETI S.L.U, Tres Cantos, Spain
}

J Investig Allergol Clin Immunol 2020; Vol. 30(2): 127-132

doi: 10.18176/jiaci.0431

\begin{abstract}
Background: Patterns of sensitization to house dust mites depend on geographic area and are important in clinical practice. However, the role of molecular diagnosis is not currently defined. We sought to characterize a pediatric population by focusing on sensitization to different mite species and major mite components in order to assess the clinical relevance of sensitization to allergenic components in our practice. Methods: Consecutive children with respiratory allergy sensitized to house dust mites (determined by skin prick test [SPT]) were recruited. We determined specific IgE to nDer p 1, rDer p 2, and rDer $p 23$ using ImmunoCAP and slgE using ImmunoCAP-ISAC microarray. Patients were followed up for 3 years.

Results: A total of 276 children were recruited. The frequency of sensitization was $86.6 \%$ for nDer $p 1,79.3 \%$ for rDer $p 2$, and $75.8 \%$ for rDer p 23. Lepidoglyphus species was the most common storage mite detected by SPT. Twenty-six patients (9.4\%) were not sensitized to Der $p 1$ or Der $p 2$. It is noteworthy that IgE binding to Der $p 23$ was positive in 14 (53.8\%). Asthmatic patients, especially those with a persistent moderate-severe phenotype, more frequently recognized the 3 major allergens.

Conclusions: Most patients with mite allergy were sensitized to the major allergens Der $p 1$, Der $p 2$, and Der $p 23$. Of the allergens evaluated, $5 \%$ were sensitized to Der $p 23$ but not to Der $p 1$ or Der $p 2$. Sensitization to Der $p 23$ should be considered in the diagnosis and treatment of mite allergy, especially in patients with moderate-severe asthma, because it may worsen the clinical phenotype.
\end{abstract}

Key words: Asthma. Component-resolved diagnosis. Der p 1. Der p 2. Der p 10. Der $p$ 23. House dust mites. Storage mites. Tropomyosin.

\section{Resumen}

Antecedentes: El perfil de sensibilización a los ácaros del polvo depende del área geográfica y es importante en la práctica clínica. Sin embargo, el papel del diagnóstico molecular no ha sido del todo definido. Nuestro objetivo fue la caracterización del perfil de sensibilización de una población pediátrica a diferentes especies de ácaros; y evaluar la sensibilización a componentes alergénicos y su relevancia en nuestra práctica clínica.

Métodos: Se reclutaron de forma consecutiva pacientes con alergia respiratoria y sensibilización a ácaros del polvo doméstico mediante pruebas cutáneas. Se determinó la IgE específica por ImmunoCAP a nDer p 1, rDer p 2, rDer p 23 y la slgE mediante el microarray de ImmunoCAP ISAC. Los pacientes fueron evaluados durante tres años según práctica cínica habitual.

Resultados: Se reclutaron un total de 276 niños. La sensibilización fue de 86,6\% a nDer p 1, 79,3\% a rDer p 2 y 75,8\% a rDer p 23. Lepidoglyphus fue el ácaro de almacén más común según prueba cutánea. Un total de veintiséis pacientes $(9,4 \%)$ no estaban sensibilizados a Der $p 1$ ni Der p 2; cabe destacar que 14 de ellos $(53,8 \%)$ presentaban IgE positiva a Der p 23. Los pacientes con asma, y en especial los de fenotipo persistente moderado y grave, reconocieron con mayor frecuencia los tres alérgenos mayores.

Conclusiones: La mayoría de nuestra población pediátrica con alergia a ácaros está sensibilizada a los alérgenos mayores Der $\mathrm{p} 1$, Der $\mathrm{p} 2$ y Der $p$ 23. Un 5\% estaba sensibilizado a Der p 23, pero no a Der $p 1$ ni a Der $p$ 2. La sensibilización a Der $p 23$ debe considerarse en el diagnóstico y tratamiento de la alergia a ácaros, especialmente en pacientes con asma persistente moderada y grave.

Palabras clave: Asma. Diagnóstico por componentes. Der $p$ 1. Der $p$ 2. Der p 10. Der p 23. Ácaros del polvo doméstico. Ácaros de almacenamiento. Tropomiosina. 


\section{Introduction}

Dust mites are the leading cause of respiratory allergy worldwide [1,2]. However, patterns of sensitization patterns to house dust mites (HDMs) and storage mites (SMs) vary depending on the geographic area [3-8]. In children, HDMs are the earliest respiratory cause of sensitization [2].

More than 30 allergens of Dermatophagoides pteronyssinus have been identified and sequenced to date [9]. Traditionally, the "major" allergens were Der p 1 and Der p 2 [7-10]. Recently, Der p 23 was identified as another major HDM allergen [11]. Nonetheless, little is known about its role in sensitization or its clinical relevance [12-18].

The efficacy of immunotherapy in patients sensitized to the major allergens of group 1 and 2 has been thoroughly demonstrated. However, in patients sensitized to other allergens, such as Der p 23, the indication for and efficacy of immunotherapy warrants further study $[4,11,15]$. Molecular diagnosis may play a role in mite allergy [14-18], although its exact utility in clinical practice has not been fully defined.

We characterized a Mediterranean pediatric population by focusing on the prevalence of sensitization to various mite species (HDM and SM) and the major components Der p 1, Der p 2, and Der $\mathrm{p} 23$. We also assessed the clinical relevance of sensitization to Der p 23 and the usefulness of commercially available allergenic components in the diagnostic work-up for mite allergy.

\section{Methods}

\section{Study Population and Study Design}

Over a 1-year period, we recruited consecutive children (318 years) with clinical manifestations of respiratory allergy from the Pediatric Allergy and Clinical Immunology Department of Hospital Sant Joan de Déu, Barcelona, Spain. The patients were sensitized to D pteronyssinus and/or Dermatophagoides farinae and had been diagnosed based on skin prick test (SPT) results. The patients were classified according to the Global Initiative for Asthma (GINA) guidelines [19] and Allergic Rhinitis and its Impact on Asthma (ARIA) guidelines [20]. If clinical criteria were fulfilled, pharmacologic treatment and allergen-specific immunotherapy were prescribed following the medical criteria of the attending specialist.

Individuals under treatment with allergen-specific immunotherapy before the study began were excluded. All patients who were under treatment were followed for at least 3 years under conditions of daily clinical practice.

We considered patients with no clinical response to immunotherapy to have uncontrolled asthma according to the GINA guidelines. These patients were characterized according to daytime symptoms, nocturnal waking due to asthma, rescue medication for relief of symptoms, and any limitation in activity after at least 3 years of treatment with immunotherapy and pharmacological treatment at the highest steps of the guidelines.

\section{Skin Prick Test}

All patients underwent SPT with biologically standardized extracts including D pteronyssinus, D farinae, and Euroglyphus maynei $(100 \mathrm{HEP} / \mathrm{mL})$, cypress pollen, olive tree pollen, grass mix pollen, Alternaria species, cat and dog epithelium $(30 \mathrm{HEP} / \mathrm{mL})$, and a battery of standardized extracts of SM, including Acarus siro, Chortoglyphus arcuatus, Glycyphagus domesticus, Lepidoglyphus destructor, Tyrophagus putrescentiae (all $30 \mathrm{HEP} / \mathrm{mL})$, and Blomia tropicalis $(100 \mathrm{HEP} / \mathrm{mL})$. Patients were also tested with purified tropomyosin from shrimp extract $(50 \mu \mathrm{g} / \mathrm{mL})[21]$.

All the SPT extracts were from Laboratorios LETI SLU, and tests were performed following EAACI guidelines [22]. A positive response was defined as the presence of a wheal with a diameter $\geq 3 \mathrm{~mm}$. Histamine chloride $10 \mathrm{mg} / \mathrm{mL}$ was used as a positive control.

\section{Serum Specific IgE}

Serum specific IgE (sIgE) to nDer $p$ 1, rDer $p$ 2, and rDer p 10 was determined in all patients $(n=276)$ by ImmunoCAP (Thermo Fisher Scientific) in accordance with the manufacturer's specifications. sIgE to rDer $\mathrm{p} 23$ was then investigated (when commercially available) in the 265 samples with sufficient volume remaining. Results over $0.35 \mathrm{kU}_{\mathrm{A}} / \mathrm{L}$ were considered positive.

SIgE to a panel of 112 allergens was also evaluated using ImmunoCAP ISAC microarray (Thermo Fisher Scientific) in all patients. The results were expressed as ISAC standardized units (ISU), with a cut-off of 0.3 ISU.

The study was approved by the local ethics committee, and signed informed consent was obtained from all patients (PIC-108-12).

\section{Statistical Analysis}

The normality of the distribution of variables was investigated using the Kolmogorov-Smirnov test. Normally and nonnormally distributed variables were expressed as mean (SD) and median (IQR), respectively.

Categorical variables were expressed as percentages and analyzed using the $\chi^{2}$ test. The Spearman correlation was used to identify linear relationships between the techniques used for molecular diagnosis. Differences were considered statistically significant if $P<.05$. Statistical analysis was performed using PASW Statistics for Windows, Version 18.0 (SPSS Inc).

\section{Results}

The study population comprised 276 prospectively and consecutively recruited children who met the inclusion criteria for respiratory allergy and sensitization to $D$ pteronyssinus and/or $D$ farinae by SPT. In $69 \%$ of cases, the patients were exclusively sensitized to HDM. The clinical characteristics of the patients are summarized in Table 1.

\section{Sensitization Profile}

Sensitization to mite extracts by skin pricks tests and the prevalence of sensitization to mite allergens by in vitro tests are summarized in Tables 2 and 3, respectively.

ImmunoCAP revealed that $90.6 \%$ of patients $(250 / 276)$ were found to be sensitized to either nDer 1 or rDer $\mathrm{p} 2$ and 
Table 1. Characteristics of the Patient Population ${ }^{a}$

\begin{tabular}{lc}
\hline Patients recruited, No. & 276 \\
Mean (SD) age/range, y & $10.32(6.7) / 3-18$ \\
Males & $85 \%$ \\
Allergic rhinoconjunctivitis, & $237(85.9 \%)$ \\
Persistent & $89(37.5 \%)$ \\
Intermittent & $148(62.5 \%)$ \\
Asthma & $191(69.2 \%)$ \\
Intermittent & $69(36 \%)$ \\
Persistent & $122(63 \%)$ \\
Mild & $49(25 \%)$ \\
Moderate & $67(35 \%)$ \\
Severe & $6(3 \%)$ \\
Immunotherapy prescription & $182(66 \%)$ \\
Completed 3 y & $140(51 \%)$ \\
Responders & $129(47 \%)$ \\
Nonresponders & $11(4 \%)$ \\
Food allergy & $32(11.6 \%)$ \\
Shellfish allergy & $4(1.4 \%)$ \\
\hline
\end{tabular}

${ }^{a}$ Data are presented as No. (\%) of patients with regard to the total $(\mathrm{N}=276)$, unless otherwise indicated.

Table 2. Prevalence of Sensitization to Mites by Skin Prick Test

\begin{tabular}{lc}
\hline Skin Prick Test & No. $(\%)$ \\
\hline Dermatophagoides pteronyssinus & $269(97.5)$ \\
Dermatophagoides farinae & $257(93.1)$ \\
Euroglyphus maynei & $252(91.3)$ \\
Lepidoglyphus destructor & $132(47.8)$ \\
Acarus siro & $132(47.8)$ \\
Chortoglyphus arcuatus & $126(45.6)$ \\
Blomia tropicalis & $85(30.8)$ \\
Glycyphagus domesticus & $56(20.3)$ \\
Tropomyosin & $18(6.5)$
\end{tabular}

that $75.8 \%(201 / 265)$ were sensitized to rDer $\mathrm{p} 23$. Of these 265 patients, $5.3 \%(n=14)$ were sensitized to rDer $\mathrm{p} 23$, but not to nDer $\mathrm{p} 1$ or rDer $\mathrm{p} 2$ and did not present a different clinical phenotype to that of those sensitized also to Der $\mathrm{p} 1 \mathrm{and} / \mathrm{or}$ Der p 2 (Table 4).

The prevalence of sensitization to Der $p$ 1, Der $p$ 2, and Der p 23 increased with age (Figure 1).

A correlation was observed between sIgE detected by ImmunoCAP and microarray for Der $\mathrm{p} 1(\mathrm{r}=0.87 ; P<.0001)$, Der p $2(\mathrm{r}=0.87 ; P<.0001)$, and Der p $10(\mathrm{r}=0.88 ; P<.0001)$ (Figure 2).

\section{Sensitization and Clinical Implications}

Major allergens were more often recognized by asthmatic patients. The prevalence of sensitization by ImmunoCAP to
Table 3. Prevalence of Sensitization to Mite Components by In Vitro Tests ${ }^{\mathrm{a}}$

\begin{tabular}{lcc}
\hline ImmunoCAP & No. $(\%)$ & Median $(\mathrm{IQR}) \mathrm{kU}_{\mathrm{A}} / \mathrm{L}$ \\
\hline nDer p 1 & $239(86.6)$ & $33.7(8.3-83.9)$ \\
rDer p 2 & $219(79.3)$ & $42.5(18.6-96.1)$ \\
rDer p 23 & $201(75.8)$ & $6.6(3.1-16.1)$ \\
rDer p 10 & $27(9.8)$ & $3.6(1.2-8.5)$ \\
\hline Microarray & $\mathrm{N}(\%)$ & Median (IQR) ISU \\
\hline nDer p 1 & $208(75.4)$ & $12(5.1-23.0)$ \\
rDer p 2 & $219(79.3)$ & $22(9.0-43.0)$ \\
nDer f 1 & $207(75.0)$ & $12(5.2-26.0)$ \\
rDer f 2 & $215(77.9)$ & $31(15.0-61.0)$ \\
rLep d 2 & $64(23.2)$ & $1.95(0.7-4.7)$ \\
rBlo t 5 & $43(15.6)$ & $1.7(0.7-4.9)$ \\
rDer p 10 & $26(9.4)$ & $10.5(2.9-28.5)$ \\
\hline
\end{tabular}

${ }^{a}$ Data are presented as No. (\%) of patients with regard to the total $(\mathrm{N}=276)$, except for rDer $p 23(\mathrm{n}=265)$.

the individual HDM allergens nDer p 1 (88.8\% vs $75.5 \%)$, rDer p 2 (83.3\% vs 70.5\%), and rDer p 23 (79\% vs 67\%) differed significantly between children with and without asthma $(P=.003, P=.01$, and $P=.02$, respectively).

Furthermore, patients with persistent moderate-severe asthma according to the GINA criteria were more frequently sensitized to Der p 23 than patients with intermittent and mild persistent forms of asthma ( $75.8 \% \mathrm{vs} 90 \%, P=.008)$.

Eleven of the patients who had been receiving immunotherapy for 3 years did not have a clinical response to allergen-specific immunotherapy, since their asthma remained uncontrolled. All of them were sensitized to Der p 23 (Table 5).

With respect to patients sensitized to Der p 23 but not to Der $\mathrm{p} 1$ or Der p $2(\mathrm{n}=14)$, only 3 had been prescribed immunotherapy, even though $50 \%$ had asthma and $71.4 \%$ persistent rhinitis. Of these 3 patients, 2 did not respond to treatment after 3 years (Table 4 ).

\section{Comparison Between SPT and Molecular Diagnosis}

All patients who were sensitized to Der $\mathrm{p} 1$ or Der $\mathrm{p} 2$ had a positive SPT result to D pteronyssinus, and $97 \%$ had a positive SPT result to $D$ farinae. Likewise, all patients sensitized exclusively to rDer p 23 had a positive SPT result to $D$ pteronyssinus, while only $50 \%$ had a positive SPT result to $D$ farinae $(P<.002)$.

Sensitization to tropomyosin by ImmunoCAP was higher than by SPT ( $9.8 \%$ vs $6.5 \%$ ). The SPT result for tropomyosin was positive for the 2 patients who had positive specific IgE to $\mathrm{rDer} \mathrm{p} 10$ without sensitization to nDer $\mathrm{p} 1$, rDer $\mathrm{p} 2$, or rDer $\mathrm{p} 23$.

Sensitization to Lepidoglyphus and Blomia species differed significantly when detected by SPT compared with microarray $(47.8 \%$ vs $23.2 \%$ and $30.8 \%$ vs $15.6 \%$, respectively; $P=.03, P=.02$ ). All patients sensitized to Blomia species according to SPT were also sensitized to major 
Table 4. Comparison of the Clinical Data of Patients Sensitized to Der p 23 Without Sensitization to Der $p 1$ and/or Der $p 2$ and Those Sensitized to Der $p 1$ or Der $p 2$ Without Sensitization to Der $p 23$

\begin{tabular}{|c|c|c|c|c|}
\hline & $\begin{array}{c}\text { Sensitized to } \\
\text { Der } \mathrm{p} 23 \text { With Der } \mathrm{p} 1 \\
\pm \text { Der } \mathrm{p} 2\end{array}$ & $\begin{array}{c}\text { Sensitized to } \\
\text { Der } \mathrm{p} 1 \pm \text { Der } \mathrm{p} 2 \\
\text { Without Sensitization to } \\
\text { Der } \mathrm{p} 23\end{array}$ & $\begin{array}{l}\text { Sensitized to Der } \mathrm{p} 23 \\
\text { Without Sensitization to } \\
\text { Der } \mathrm{p} 1 \pm \text { Der } \mathrm{p} 2\end{array}$ & $P$ Value \\
\hline No. & 183 & 79 & 14 & \\
\hline Mean (SD) age, y & $10(2)$ & $10(5)$ & $11(3)$ & .37 \\
\hline Median (IQR) SPT DP, mm² $^{2}$ & $25(10-45)$ & $23(12-35)$ & $27(18-55.5)$ & .53 \\
\hline Median (IQR) SPT DF, $\mathrm{mm}^{2}$ & $32(12-32)$ & $36(37-46)$ & $30(18-48)$ & .16 \\
\hline $\begin{array}{l}\text { Asthma, No. }(\%) \\
\text { Intermittent } \\
\text { Mild } \\
\text { Moderate } \\
\text { Severe }\end{array}$ & $\begin{array}{c}132(70 \%) \\
36(27 \%) \\
32(24.5 \%) \\
58(44 \%) \\
6(4.5 \%)\end{array}$ & $\begin{array}{c}36(45.5 \%) \\
25(69.5 \%) \\
5(14 \%) \\
6(16.5 \%) \\
0(0 \%)\end{array}$ & $\begin{array}{l}7(50 \%) \\
3(43 \%) \\
1(14 \%) \\
3(43 \%) \\
0(0 \%)\end{array}$ & $\begin{array}{c}<.0001 \\
.2 \\
.06 \\
<.0001 \\
.69\end{array}$ \\
\hline Rhinitis, No. (\%) & $150(82 \%)$ & $27(69 \%)$ & $10(71 \%)$ & .73 \\
\hline
\end{tabular}

Abbreviations: DP, Dermatophagoides pteronyssinus; DF, Dermatophagoides farinae; SPT, skin prick test.

components of Dermatophagoides. As for patients sensitized to Lepidoglyphus, only 1 patient reacted to rLep d 2 without sensitization to Der p 1, Der p 2, or Der p 23.

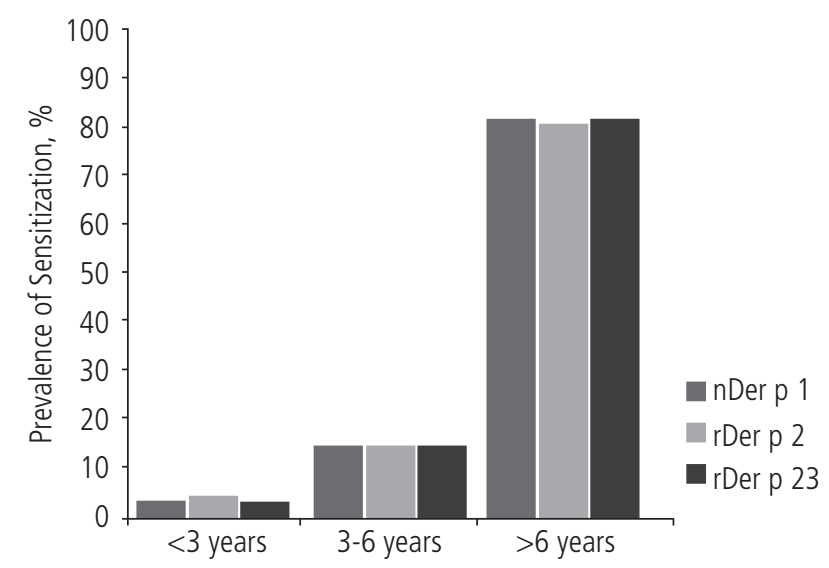

Figure 1. Prevalence of sensitization to nDer $\mathrm{p} \mathrm{1,} \mathrm{rDer} \mathrm{p} 2$, and rDer $\mathrm{p} 23$ by age groups (detected by ImmunoCAP).

\section{Discussion}

This study describes the profile of sensitization to different HDM and SM species and sensitization to major allergens in a pediatric population with respiratory allergy disease. It also illustrates the clinical relevance of sensitization to Der p 23. For the first time in children, sensitization to Der $\mathrm{p} 23$ has been linked to persistent moderate-severe asthma.

To date, the diagnostic value of combining molecular diagnosis with SPT has not been fully established. Our study proposes criteria for the real-life application of molecular diagnosis in patients with respiratory allergy and positive SPT results to mites.

As expected, Der $p 1$ and/or Der $\mathrm{p} 2$ were recognized by most patients (90.6\%), and Der p 23, which has been identified as a major HDM allergen [7,8,11-15,18,23-25], was recognized by $75.8 \%$ of HDM-allergic patients. This finding is consistent with data from other Mediterranean series [10,12-14,24,26]. Remarkably, in $9.4 \%$ of patients in the present study, no sensitization to the major allergens Der $\mathrm{p} 1$ and Der $\mathrm{p} 2$ was detected. It is important to note that $56 \%$ of these patients were sensitized to Der p 23. n Der p 1

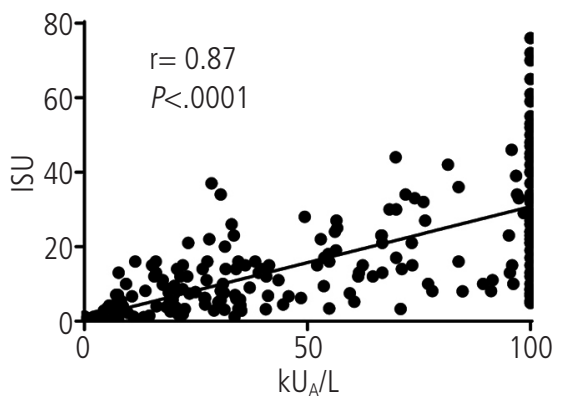

n Der $\mathrm{p} 2$

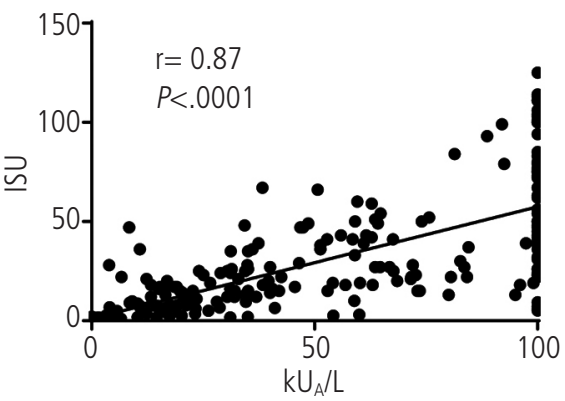

n Der p 10

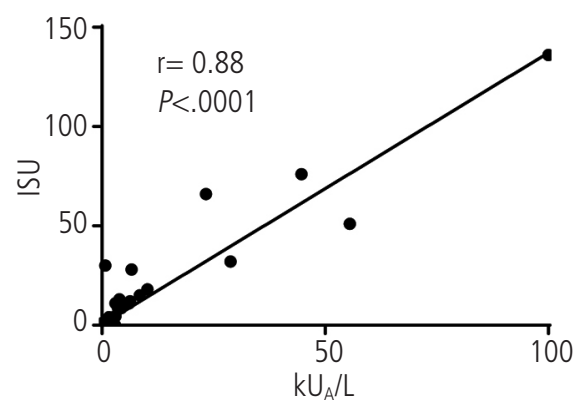

Figure 2. Correlation between levels of specific lgE to nDer p 1, rDer p2, and rDer $p 10$ determined by ImmunoCAP ( $\left.\mathrm{KU} \mathrm{A}_{\mathrm{A}} / \mathrm{L}\right)$ or ISAC (ISU). 
Table 5. Comparison of the Clinical Data of Patients With a Clinical Response to Immunotherapy and Patients Without a Response to Immunotherapya

\begin{tabular}{|c|c|c|c|}
\hline & $\begin{array}{l}\text { Responders to } \\
\text { Immunotherapy }\end{array}$ & $\begin{array}{l}\text { Nonresponders to } \\
\text { Immunotherapy }\end{array}$ & $P$ Value \\
\hline No. $(\%)$ & $129 / 140(92 \%)$ & $11 / 140(8 \%)$ & \\
\hline $\begin{array}{l}\text { Mean (SD) age, y } \\
\text { Sensitization to nDer p } 1 \\
\text { Sensitization to rDer p } 2 \\
\text { Sensitization to rDer p } 23\end{array}$ & $\begin{array}{c}10(3) \\
116(90 \%) \\
109(87.2 \%) \\
99(79.2 \%)\end{array}$ & $\begin{array}{c}15(2) \\
11(100 \%) \\
11(100 \%) \\
10(91 \%)\end{array}$ & $\begin{array}{l}.53 \\
.56 \\
.46 \\
.24\end{array}$ \\
\hline $\begin{array}{l}\text { Sensitized to nDer } \mathrm{p} 1 \pm \\
\text { rDer } \mathrm{p} 2 \text { with sensitization to rDer } \mathrm{p} 23\end{array}$ & $12(9.3 \%)$ & $1(9 \%)$ & .12 \\
\hline $\begin{array}{l}\text { Sensitized to rDer } \mathrm{p} 23 \\
\text { without sensitization nDer } \mathrm{p} 1 \pm \text { rDer } \mathrm{p} 2\end{array}$ & $1(0.7 \%)$ & $2(18 \%)$ & .12 \\
\hline Median (IQR) IgE to $\mathrm{nDer} p 1 \mathrm{kU}_{\mathrm{A}} / \mathrm{L}$ & $34(10-93)$ & $34(1.5-100)$ & .73 \\
\hline Median (IQR) IgE to rDer p $2 \mathrm{kU}_{\mathrm{A}} / \mathrm{L}$ & $38(0.5-98.5)$ & $24(0.5-92)$ & .36 \\
\hline Median (IQR) IgE to rDer p $23 \mathrm{kU}_{\mathrm{A}} / \mathrm{L}$ & $6(1-14)$ & $11(3-22)$ & .16 \\
\hline
\end{tabular}

aData Are Presented as No. (\%), unless otherwise indicated $(\mathrm{N}=140)$.

Interestingly, sensitization to group 1 major allergens was more prevalent than sensitization to group 2 major allergens. We found that the prevalence of asthma was almost 70\% (63\% persistent asthma) and that Der p 1 was associated with the development and severity of asthma in childhood [20,23].

Our study revealed greater recognition of Der $\mathrm{p} 1$, Der p 2, and Der p 23 in asthmatic patients than in those with rhinitis. This finding agrees with those of other investigations $[9,11,13,15]$. An important finding in our study was the significantly higher recognition of Der $\mathrm{p} 23$ in patients with persistent moderate-severe asthma.

Furthermore, most patients with sensitization to Der p 23, but not to Der $\mathrm{p} 1$ or Der $\mathrm{p} 2$, had not received immunotherapy, even when they could have potentially benefited from it. Moreover, the 11 patients who did not respond to immunotherapy were all sensitized to Der p 23.

Therefore, Der p 23 was an important component that should be included in the diagnosis of patients with respiratory allergy, as well as a possible tool for improving the patient's clinical course.

In our study, $9.8 \%$ of patients were sensitized to tropomyosin, consistent with another study of children from a Mediterranean area [20]. As expected, most children sensitized to Der p 10 were also sensitized to the major allergens tested. Only $2(0.7 \%)$ were sensitized to Der p 10 without being sensitized to Der $\mathrm{p} \mathrm{1,} \mathrm{Der} \mathrm{p} 2$, or Der $\mathrm{p} 23$, thus confirming that Der p 10 is a minor allergen in respiratory allergy to mites [19-22]. These children had a positive SPT result to tropomyosin. Thus, the inclusion of purified shrimp tropomyosin in the panel for diagnosis of respiratory allergy could be relevant. Moreover, Resch et al [10] recently concluded that Der p 10-positive patients reacted to the other mite allergens $\mathrm{rDer} p 5$, rDer $\mathrm{p} 7$, and rDer $\mathrm{p}$ 21. Therefore, patients whose SPT yielded positive results to tropomyosin could be interesting candidates for further molecular diagnosis.
Finally, we observed a high rate of sensitization to SM allergens. A key limitation of our study was that we studied sensitization to SM in patients who were selected based on positive SPT results to HDM. Therefore, we missed patients who were exclusively sensitized to SM, although in our area, sensitization to SM only has previously been reported to be low $(0.8 \%)$ [6]. Lepidoglyphus was considered the predominant SM species in Europe, followed by Blomia. The prevalence of sensitization to Lep d 2 and Blo t 5 was lower than the frequency of sensitization detected with the corresponding extracts by SPT. This discrepancy may indicate sensitization to other components that were not represented in the molecular diagnosis platform used. Therefore, it is important to determine sensitization to both the whole extract and to the major allergens Lep d 2 and Blo 5 before prescription of SM-specific immunotherapy.

It is also remarkable that all children sensitized to Lepidoglyphus, except 1, and all children sensitized to Blomia were also sensitized to the major allergens of $D$ pteronyssinus and $D$ farinae. This is in accordance with previous findings [4,25], which state that sensitization to SM is related to exposure to HDM.

In conclusion, this study confirms the importance of molecular components in aiding further diagnosis in miteallergic patients. We demonstrate the importance of major allergens in patients with respiratory allergy to mites and the implications of Der $\mathrm{p} 23$ for clinical practice, as this allergen is more prevalent in persistent moderate-severe asthma in children.

The high frequency of sensitization to Der p 23 detected suggests the need to include this allergen in diagnostic and therapeutic allergen preparations. Further studies are required to consolidate these observations.

\section{Funding}

The authors declare that no funding was received for the present study. 


\section{Conflicts of Interest}

Moya R and Carnés J were employed by LETI Laboratories at the time of the study and continue to be employees of the company. Their employment entails no direct conflicts with the data presented in this study. The remaining authors declare that they have no conflicts of interest.

\section{References}

1. Fernández-Caldas $\mathrm{E}$, Iraola V. Mite allergens. Curr Allergy Asthma Rep. 2005;5:402-10.

2. The International Study of Asthma and Allergies in Childhood (ISAAC). Available at: http://isaac.auckland.ac.nz. Accessed May 21, 2018

3. Cui YB, Yu LL, Teng FX, Wang N, Zhou Y, Yang L, et al. Dust mite allergen Der $\mathrm{f} 4$ : Expression, characterization, and $\lg E$ binding in pediatric asthma. Pediatr Allergy Immunol. 2016;27(4):391-7.

4. Pittner G, Vrtala S, Thomas WR, Weghofer M, Kundi M, Horak $F$, et al. Component-resolved diagnosis of house-dust mite allergy with purified natural and recombinant mite allergens. Clin Exp Allergy. 2004;34:597-603.

5. Morales M, Iraola V, Leonor JR, Bartra J, Rodríguez F, Boquete $M$, et al. Different sensitization to storage mites depending on the co-exposure to house dust mites. Ann Allergy Asthma Immunol. 2015;114:36-42.

6. Gaig P, Botey J, Pena M, Marin A, Eseverri JL. Study of the sensitization to storage mites in a pediatric population in Barcelona. J Invest Allergol Clin Immunol. 1993;3:151-5.

7. Weghofer M, Thomas WR, Kronqvist M, Mari A, Purohit A, Pauli $G$, et al. Variability of IgE reactivity profiles among European mite allergic patients. Eur J Clin Invest. 2008;38(12):959-65.

8. Iraola V, Boquete M, Pinto H, Carballada, Caballas C, Carnes J. Pattern of sensitization to Major allergens Der $p 1$ and Der $p$ 2 in mite sensitized individuals from Galicia, Spain. J Investig Allergol Clin Immunol. 2010;20(3):267-72.

9. WHO/IUIS Allergen Nomenclature. Available at: http://www. allergen.org/. Ac-cessed May 21, 2018.

10. Resch $Y$, Weghofer M, Seiberler S, Horak F, Scheiblhofer $S$, Linhart B, et al. Molecular characterization of Derp 10: a diagnostic marker for broad sensitization in house dust mite allergy. Clin Exp Allergy. 2011;41(10):1468-77.

11. Weghofer M, Grote M, Resch Y, Casset A, Kneidinger M, Kopec J, et al. Identification of Der p 23, a peritrophin-like protein, as a new major Dermatophagoides pteronyssinus allergen associated with the peritrophic matrix of mite fecal pellets. J Immunol. 2013;190(7):3059-67.

12. Banerjee S, Weber M, Blatt K, Swoboda I, Focke-Tejkl M, Valent $\mathrm{P}$, et al. Conversion of Der $\mathrm{p}$ 23, a new major house dust mite allergen, into a hypoallergenic vaccine. J Immunol. 2014;192(10):4867-75.

13. Posa D, Perna S, Resch Y, Lupinek C, Panetta V, Hofmaier $S$, et al. Evolution and predictive value of IgE responses toward a comprehensive panel of house dust mite allergens during the first 2 decades of life. J Allergy Clin Immunol. 2017;139:541-9.

14. Resch Y, Michel S, Kabesch M, Lupinek C, Valenta R, Vrtala $S$. Different lgE recognition of mite allergen components in asthmatic and non-asthmatic children. J Allergy Clin Immunol. 2015;136(4):1038-91.
15. Mueller GA, Randall TA, Glesner J, Pedersen LC, Perera $L$, Edwards LL, et al. Serological, genomic and structural analyses of the major mite allergen Der p 23. Clin Exp Allergy. 2016;46(2):365-76.

16. Sylvestre L, Jegu J, Metz-Favre C, Barnig C, Qi S, de Blay F. Component-Based Allergen Microarray: Der p 2 and Der $f$ Dust Mite sensitization is more common in patients with severe asthma. J Investig Allergol Clin Immunol. 2016;26(2):141-3.

17. Vidal C, Lojo S, Juangorena M, Gonzalez-Quintela A Association between asthma and sensitization to allergens of Dermatophagoides pteronyssinus. J Investig Allergol Clin Immunol. 2016;26(5):304-9.

18. Matos Semedo F, Dorofeeva Y, Pires AP, Tomaz E, Taborda Barata L, Inacio F, et al. Der p 23-clinical relevance of molecular monosensitisation in house dust mite allergy. J Investig Allergol Clin Immunol. 2019;29(4):314-6.

19. The Global Initiative for Asthma (GINA) Report. Global strategy for asthma man-agement and prevention (updated 2016).

20. Bousquet J, Khaltaev N, Cruz AA, Denburg J, Fokkens WJ, Togias A, et al. Allergic Rhinitis and its Impact on Asthma (ARIA) 2008 update (in collaboration with the world Health Organization, GA(2)LEN and AllerGen). Allergy. 2008;63 Suppl 86:8-160.

21. Matricardi PM, Kleine-Tebbe J, Hoffmann HJ, Valenta $R$ Hilger C, Hofmaier $\mathrm{S}$, et al. In vivo diagnosis with purified tropomyosin in mite and shellfish allergic patients. Ann Allergy Asthma Immunol. 2016;116(6):538-43.

22. Van Kampen $V$, de Baly F, Folleti I, Kobiersi P, Mocato G, Olivieri $M$, at al. EAACl position paper: skin prick testing in the diagnosis of occupational type 1 allergies. Allergy. 2013:68:580-4

23. Sporik R. Holgate ST, Platts- Mills TA, Cogswell JJ. Exposure to house-dust mite allergen (Der p 1) and the development of asthma in childhood. A prospective study. N Engl J Med. 1990;323(8):502-7.

24. Batard T, Baron-Bodo V, Martelet $A$, Le Mignon M, Lemoine $P_{\text {, }}$ Jain $\mathrm{K}$, et al. Pat-terns of IgE sensitization in house dust miteallergic patients: implications for allergen immunotherapy. Allergy. 2016;71(2):220-9.

25. Barber D, Arias J, Boquete M, Cardona V, Carrillo T, Gala G, et al. Analysis of mite allergic patients in a diverse territory by improved diagnostic tools. Clin Exp Allergy. 2012;42(7):1129-38.

26. Arroabarren E, Echechipia S, Galbete A, Lisazo MT, Olaguibel JM, Tabar Al. Association between component-resolved diagnosis of house dust mite and efficacy and safety of specific immunotherapy. J Investig Allergol Clin Immunol. 2019;29(2):164-7.

Manuscript received February 27, 2019; accepted for publication June 27, 2019.

\section{- Rosa M Jiménez-Feijoó}

Department of Pediatric Allergy and Clinical Immunology Hospital Sant Joan de Déu

Universitat de Barcelona

C/ Sant Joan de Déu, 2

08950 Esplugues de Llobregat (Barcelona), Spain

E-mail: rjimenezf@sjdhospitalbarcelona.org 